\title{
REGULARITY FOR ENTROPY SOLUTIONS OF PARABOLIC $p$-LAPLACIAN TYPE EQUATIONS
}

\author{
S. Segura de León and J. Toledo
}

\begin{abstract}
In this note we give some summability results for entropy solutions of the nonlinear parabolic equation $u_{t}-\operatorname{div} \mathbf{a}_{p}(x, \nabla u)=f$ in $] 0, T\left[\times \Omega\right.$ with initial datum in $L^{1}(\Omega)$ and assuming Dirichlet's boundary condition, where $\mathbf{a}_{p}(.,$.$) is a Carathéodory function sat-$ isfying the classical Leray-Lions hypotheses, $f \in L^{1}(] 0, T[\times \Omega)$ and $\Omega$ is a domain in $\mathbb{R}^{N}$. We find spaces of type $L^{r}\left(0, T ; \mathcal{M}^{q}(\Omega)\right)$ containing the entropy solution and its gradient. We also include some summability results when $f=0$ and the $p$-Laplacian equation is considered.
\end{abstract}

\section{Introduction}

Let $\Omega$ be a domain in $\mathbb{R}^{N}$ (bounded or not) and let $1<p<N$. Let $\mathbf{a}_{p}: \Omega \times \mathbb{R}^{N} \rightarrow \mathbb{R}^{N}$ be a Carathéodory function satisfying the classical Leray-Lions conditions in such a way that $\operatorname{div} \mathbf{a}_{p}(x, \nabla u(x))$ defines an operator from $W_{0}^{1, p}(\Omega)$ onto $W^{-1, p^{\prime}}(\Omega)$ (see [10]). The model example of such function is $\mathbf{a}_{p}(x, \xi)=|\xi|^{p-2} \xi$ which determines the $p$-Laplacian operator $\Delta_{p} u=\operatorname{div}\left(|\nabla u|^{p-2} \nabla u\right)$.

1991 Mathematics subject classifications: 35K65, 47H20.

This research has been supported by the Spanish DGICYT, Proyecto PB94-0960. 
We are interested in the regularity of entropy solutions (see Definition 2.4 below) of the following parabolic problem:

$$
\begin{cases}u_{t}-\operatorname{div} \mathbf{a}_{p}(x, \nabla u)=f, & \text { in } \left.Q_{T}:=\right] 0, T[\times \Omega, \\ u=0, & \text { on } \left.S_{T}:=\right] 0, T[\times \partial \Omega, \\ u(0)=u_{0}, & \text { in } \Omega ;\end{cases}
$$

where $u_{0} \in L^{1}(\Omega)$ and $f \in L^{1}\left(Q_{T}\right)$.

A distributional solution of this problem was found in $[\mathbf{9}]$. The notion of entropy solution of $(\mathrm{P})$ is used in $[\mathbf{1}]$ and $[\mathbf{1 1}]$ to obtain uniqueness. (An equivalent concept, in terms of renormalized solution, can be found in $[\mathbf{7}],[\mathbf{1 3}],[\mathbf{1 4}]$ and $[\mathbf{1 6}]$.) We remark that in $[\mathbf{1}]$ it is only considered the case $f=0$, although the proof of the general case can be easily obtained following the same steps and taking into account the arguments of $[\mathbf{6}$, Chapter 4]. On the other hand, the hypotheses in [11] are $p>2-\frac{1}{N+1}$ and $\Omega$ bounded. In both articles some summability results on the entropy solution $u$ and its gradient are given; more precisely, $u \in \mathcal{M}^{\frac{(N+1) p-N}{N}}\left(Q_{T}\right)$ and $|\nabla u| \in \mathcal{M}^{p-\frac{N}{N+1}}\left(Q_{T}\right)$ (see [1] ), and $|\nabla u| \in \cap_{q<p-\frac{N}{N+1}} L^{q}\left(Q_{T}\right)$ (see $[\mathbf{1 1}]$ or $[\mathbf{9}])$. These summability results are obtained jointly for time and space variables; thus, as a consequence of these papers, it is not possible to get optimal regularity when both variables are separately considered.

The purpose of this note is to give a precise summability result of the entropy solution and its gradient with respect to space and time. This was studied in $[8]$ for weak solutions of $(\mathrm{P})$ in the framework of Lebesgue and Sobolev spaces when $p \geq 2, u_{0}=0$ and $\Omega$ is bounded. The main result of $[8]$ states that there exists a weak solution $u$ of $(\mathrm{P})$ such that $u \in L^{r}\left(0, T ; W_{0}^{1, q}(\Omega)\right)$ for $p / 2 \leq q<N(p-1) /(N-1)$ and $1 \leq r<q((N+1) p-2 N) /((N+1) q-N)$, and for $1 \leq q<p / 2$ and $r=p$.

Some remarks on their assumptions are as follows. Firstly, it is possible to get a similar result if $2-1 /(N+1)<p<2$, as it is observed in [8, Remark 1.7]. Moreover, analogous results also hold for other initial data $u_{0} \neq 0$, as it is pointed out in $[\mathbf{1 2}]$. Nevertheless, their hypothesis of $\Omega$ bounded cannot be removed if their arguments are to be followed, in fact it is needed from the very begining when they obtain the a priori estimates in [8, Lemma 2.2]. We want to show in this paper what the situation looks like in the framework of Marcinkiewicz spaces (see Definition 2.1 below) since we think they should be more suitable for $L^{1}$-data (see $[\mathbf{3}]$ for the elliptic case). We obtain an improvement of the results of $[\mathbf{8}]$ since our arguments work for small $p>1$ and unbounded domains. 
Theorem 1. Let $\Omega$ be a bounded domain and let $u$ be the entropy solution of problem $(P)$.

(1) If $\frac{2 N}{N+1}<p<N$, then

$$
\begin{aligned}
& u \in L^{r}\left(0, T ; \mathcal{M}^{q}(\Omega)\right) \quad \text { for } 1<q< \frac{N(p-1)}{N-p} \\
& \text { and } r<\frac{(N+1) p-2 N}{N} \frac{q}{q-1}, \\
&|\nabla u| \in L^{r}\left(0, T ; \mathcal{M}^{q}(\Omega)\right) \quad \text { for } \frac{p}{2}<q< \frac{N(p-1)}{N-1} \\
& \text { and } r<q \frac{(N+1) p-2 N}{(N+1) q-N}, \\
& \text { for } q=\frac{p}{2} \quad \text { and } r<p, \quad \text { and } \\
& \text { for } q<\frac{p}{2} \quad \text { and } r=p .
\end{aligned}
$$

(2) If $1<p \leq \frac{2 N}{N+1}$, then

$|\nabla u| \in L^{p}\left(0, T ; \mathcal{M}^{q}(\Omega)\right) \quad$ for $q<\frac{p}{2}$.

Remark 1.1. When $\Omega$ is bounded, one can replace $\mathcal{M}^{q}(\Omega)$ by $L^{q}(\Omega)$ due to the relations between Marcinkiewicz and $L^{q}$ spaces in bounded domains, and to the fact that $q$ satisfies strict inequalities.

Theorem 2. Let $\Omega$ be an unbounded domain and let $u$ be the entropy solution of problem $(P)$.

(1) If $\frac{2 N}{N+1}<p<N$, then

$$
\begin{aligned}
u \in L^{r}\left(0, T ; \mathcal{M}^{q}(\Omega)\right) \quad \text { for } 1<q< & \frac{N(p-1)}{N-p} \\
& \text { and } r<\frac{(N+1) p-2 N}{N} \frac{q}{q-1}, \\
|\nabla u| \in L^{r}\left(0, T ; \mathcal{M}^{q}(\Omega)\right) \quad \text { for } \frac{p}{2}<q< & \frac{N(p-1)}{N-1} \\
& \text { and } r<q \frac{(N+1) p-2 N}{(N+1) q-N} .
\end{aligned}
$$


(2) If $1<p<\frac{2 N}{N+1}$, then

$$
\begin{aligned}
u \in L^{r}\left(0, T ; \mathcal{M}^{q}(\Omega)\right) \quad \text { for } \frac{N(p-1)}{N-p}<q & <1 \\
\text { and } r & <\frac{(N+1) p-2 N}{N} \frac{q}{q-1}, \\
|\nabla u| \in L^{r}\left(0, T ; \mathcal{M}^{q}(\Omega)\right) \quad \text { for } \frac{N(p-1)}{N-1}<q & <\frac{p}{2} \\
\text { and } r & <q \frac{(N+1) p-2 N}{(N+1) q-N} .
\end{aligned}
$$

Remark 1.2. For $p=2$, the bound on $r$ is exactly the same one that can be computed for the fundamental solution of the Heat equation.

We improve these regularity results when $f=0$ and the $p$-Laplacian operator is considered.

Theorem 3. Let $\Omega$ be a bounded or unbounded domain, $1<p<N$ and let $u$ be the entropy solution of problem $(P)$ with the p-Laplacian operator and $f=0$, then

$$
\begin{aligned}
u & \in L^{r}\left(0, T ; \mathcal{M}^{\frac{N(p-1)}{N-p}}(\Omega)\right) & & \text { for } r<p-1 . \\
|\nabla u| & \in L^{r}\left(0, T ; \mathcal{M}^{\frac{N(p-1)}{N-1}}(\Omega)\right) & & \text { for } r<p-1 . \\
|\nabla u| & \in L^{r}\left(0, T ; \mathcal{M}^{\frac{p}{2}}(\Omega)\right) & & \text { for } r<p .
\end{aligned}
$$

This paper is divided into five sections. The next one is on preliminaries: we include the definitions of Marcinkiewicz spaces and entropy solutions of problem $(\mathrm{P})$ and the first estimates on the solution and its derivative. Section 3 is devoted to prove Theorem 1 (Theorems 3.1 and 3.2 ), while in section 4 we prove Theorem 2 (Theorems 4.1 and 4.2). Finally, in section 5 , we prove Theorem 3 , and we also give other regularity results when $f=0$ and the initial datum $u_{0}$ lies in $\mathcal{M}^{s}(\Omega) \cap L^{1}(\Omega)$ or $L^{s}(\Omega) \cap L^{1}(\Omega), 1<s<2$. 


\section{Preliminaries}

Throughout this note the Lebesgue measure on $\Omega$ will be denoted by $\mu_{N}$.

Definition 2.1. For $0<q<\infty$, the set of all measurable functions $u$ : $\Omega \rightarrow \mathbb{R}$ such that the functional $[u]_{q}:=\sup _{k>0} k \mu_{N}\{|u|>k\}^{1 / q}$ is finite is called a Marcinkiewicz space and is denoted by $\mathcal{M}^{q}(\Omega)$.

It is straightforward that, for bounded $\Omega$, we have $\mathcal{M}^{q}(\Omega) \subset \mathcal{M}^{r}(\Omega)$ for $r<q$. The connection between Marcinkiewicz and Lebesgue spaces is easy: $L^{q}(\Omega) \subset \mathcal{M}^{q}(\Omega) \subset L_{\text {loc }}^{r}(\Omega)$ for $r<q$ (see, for instance, [17]); let us point out that Marcinkiewicz spaces are also known as weak-Lebesgue spaces.

When $q>1$, the Marcinkiewicz space $\mathcal{M}^{q}(\Omega)$ is a Banach space with the norm defined by $\|u\|_{q}=\sup _{t>0} t^{\frac{1-q}{q}} \int_{0}^{t} u^{*}(\tau) d \tau$, where $u^{*}(\tau)=$ $\inf \left\{k>0: \mu_{N}\{|u|>k\} \leq \tau\right\}$ defines the non-increasing rearrangement of $u$ (see, for instance, [17, Definition 1.8.6]). As a consequence of this definition one has that $\int_{K}|u| \leq \mu_{N}\{K\}^{\frac{q-1}{q}}\|u\|_{q}$ for any $K \subset \Omega$ with finite measure. Note that, endowed with this norm, if $u, v \in \mathcal{M}^{q}(\Omega)$, then $\int_{0}^{t} u^{*}(\tau) d \tau \leq \int_{0}^{t} v^{*}(\tau) d \tau$ for all $t>0$ implies $\|u\|_{q} \leq\|v\|_{q}$; that is, $\mathcal{M}^{q}(\Omega)$ is a normal space in the sense of [5, Definition 2.8].

Definition 2.2. If $r, q \in] 0,+\infty[$, we will say that a measurable function $u:] 0, T\left[\times \Omega \rightarrow \mathbb{R}\right.$ belongs to $L^{r}\left(0, T ; \mathcal{M}^{q}(\Omega)\right)$ if $\int_{0}^{T}[u(t)]_{q}^{r} d t$ is finite.

The following result is well known; a proof may be seen, for instance, in [2, Lemma 1.3].

Lemma 2.3. Let $I \subset \mathbb{R}$ be an interval and denote by $\Lambda$ the set of all measurable functions $\lambda:[0, T] \rightarrow I$. Let $f:[0, T] \times I \rightarrow] 0,+\infty[$ be a function such that for each $\lambda \in \Lambda$ the function $t \rightarrow f(t, \lambda(t))$ is measurable. Then $\int_{0}^{T}\left(\sup _{k \in I} f(t, k)\right) d t=\sup _{\lambda \in \Lambda} \int_{0}^{T} f(t, \lambda(t)) d t$.

Before introducing the concept of entropy solution, we will define the following functional spaces (see $[\mathbf{3}]$ ). Given $k>0$, define the truncature operator by $T_{k}(\zeta)=(\zeta \wedge k) \vee(-k)$, whose primitive is $J_{k}(\xi)=\int_{0}^{\xi} T_{k}(s) d s$. The space of all measurable functions $u: \Omega \rightarrow \mathbb{R}$ such that $T_{k} u \in$ $W_{\text {loc }}^{1,1}(\Omega)$ and $\nabla T_{k} u \in L^{p}(\Omega)$ for all $k>0$ is denoted by $\mathcal{T}^{1, p}(\Omega)$ while $\mathcal{T}_{0}^{1, p}(\Omega)$ denotes the space of all functions $u \in \mathcal{T}^{1, p}(\Omega)$ such that for every $k>0$ there exists a sequence $\left(\phi_{n}\right)$ in $C_{0}^{\infty}(\Omega)$ satisfying $\nabla \phi_{n} \rightarrow \nabla T_{k} u$ in 
$L^{p}(\Omega)$ and $\phi_{n} \rightarrow T_{k} u$ in $L_{\text {loc }}^{1}(\Omega)$. If $u \in \mathcal{T}^{1, p}(\Omega)$, a derivative $\nabla u$ can be defined as the unique measurable function such that $\nabla T_{k} u=\nabla u \cdot \chi_{\{|u|<k\}}$ for all $k>0$ (see [3, Lemma 2.1]).

Definition 2.4. We will say that a function $u \in C\left([0, T] ; L^{1}(\Omega)\right)$ is an entropy solution of $(\mathrm{P})$ if $u(t) \in \mathcal{T}_{0}^{1, p}(\Omega)$ for almost all $t, \nabla T_{k}(u) \in$ $L^{p}\left(Q_{T}\right)$ for all $k>0$ and

$$
\begin{aligned}
& \int_{0}^{t} \int_{\Omega} \varphi^{\prime} T_{k}(u-\varphi)+\int_{0}^{t} \int_{\Omega}\left\langle\mathbf{a}_{p}(x, \nabla u), \nabla T_{k}(u-\varphi)\right\rangle \\
& \quad \leq \int_{\Omega} J_{k}\left(u_{0}-\varphi(0)\right)-\int_{\Omega} J_{k}(u(t)-\varphi(t))+\int_{0}^{t} \int_{\Omega} f T_{k}(u-\varphi)
\end{aligned}
$$

for all $k>0, t \in[0, T]$ and $\varphi \in L^{\infty}\left(Q_{T}\right) \cap L^{p}\left(0, T ; W_{0}^{1, p}(\Omega)\right) \cap$ $W^{1,1}\left(0, T ; L^{1}(\Omega)\right)$.

Taking $\varphi=0$ in the formulation of entropy solution it follows that

$$
\begin{aligned}
\frac{1}{k} \int_{0}^{T} \int_{\Omega}\left\langle\mathbf{a}_{p}(x,\right. & \left.\nabla u), \nabla T_{k} u\right\rangle \\
& \leq \int_{\Omega} \frac{J_{k}\left(u_{0}\right)}{k}+\int_{0}^{T} \int_{\Omega} f \frac{T_{k} u}{k} \leq \int_{\Omega}\left|u_{0}\right|+\int_{0}^{T} \int_{\Omega}|f|
\end{aligned}
$$

for all $k>0$. Consequently,

$$
\int_{0}^{T} \int_{\Omega} \frac{\left|\nabla T_{k} u(t)\right|^{p}}{k} \leq M, \quad \forall k>0
$$

being $M=\int_{\Omega}\left|u_{0}\right|+\int_{0}^{T} \int_{\Omega}|f|$. (Recall that one of the Leray-Lions assumptions asserts that there exists $\alpha>0$ satisfying $\alpha|\xi|^{p} \leq\left\langle\mathbf{a}_{p}(x, \xi), \xi\right\rangle$; we consider $\alpha=1$ since there is not loss of generality in doing so.)

From now on $u$ will denote the entropy solution of $(\mathrm{P})$.

We will finish this section giving the basic estimates in order to prove our regularity results. We will use the fact that $u \in C\left([0, T], L^{1}(\Omega)\right)$ and the estimate given in (2.1).

Proposition 2.5. For every $\delta>0$ there is $g \in L^{1}(0, T)$ such that

$$
\int_{\Omega} \frac{\left|\nabla T_{k} u(t)\right|^{p}}{k}= \begin{cases}k^{\delta} g(t), & \text { if } t \in[0, T] \text { and } k \geq 1 \\ k^{-\delta} g(t), & \text { if } t \in[0, T] \text { and } k \leq 1\end{cases}
$$


Proof: We just prove the first assertion, the second one can be proved similarly. Consider a measurable function $\lambda:[0, T] \rightarrow[1,+\infty[$ and the sets $A_{j}:=\left\{t \in[0, T]: 2^{j} \leq \lambda(t)<2^{j+1}\right\}, j \geq 0$, and compute to get the following inequalities

$$
\begin{aligned}
\int_{0}^{T} \int_{\Omega} \frac{\left|\nabla T_{\lambda(t)} u(t)\right|^{p}}{\lambda(t)^{1+\delta}} & \leq \sum_{j=0}^{\infty} \int_{A_{j}} \int_{\Omega} \frac{\left|\nabla T_{2^{j+1}} u(t)\right|^{p}}{2^{j(1+\delta)}} \\
& =\sum_{j=0}^{\infty} \frac{2}{2^{j \delta}} \int_{A_{j}} \int_{\Omega} \frac{\left|\nabla T_{2^{j+1}} u(t)\right|^{p}}{2^{j+1}} \leq 2 M \sum_{j=0}^{\infty} \frac{1}{2^{j \delta}} .
\end{aligned}
$$

By Lemma 2.3,

$$
\int_{0}^{T} \sup _{k \geq 1} \int_{\Omega} \frac{\left|\nabla T_{k} u\right|^{p}}{k^{1+\delta}}<\infty
$$

and now Fubini's theorem allows us to take $g(t) \geq \sup _{k \geq 1} \int_{\Omega} \frac{\left|\nabla T_{k} u(t)\right|^{p}}{k^{1+\delta}}$.

Observe that there is no loss of generality in taking $g(t) \geq M^{\frac{N-p}{N}}$ as we will do in our following estimates.

Proposition 2.6. Let $\delta \in] 0, p-1[$. Then there exists a constant $C>0$ such that for almost all $t \in[0, T]$,

$$
\begin{aligned}
& \sup _{k \geq 1} k \mu_{N}\{|u(t)|>k\}^{\frac{N-p}{N(p-1-\delta)}} \leq(C g(t))^{\frac{1}{p-1-\delta}} \\
& \sup _{k \leq 1} k \mu_{N}\{|u(t)|>k\}^{\frac{N-p}{N(p-1+\delta)}} \leq(C g(t))^{\frac{1}{p-1+\delta}} .
\end{aligned}
$$

Proof: Proposition 2.5 and Sobolev's inequality imply two facts: on the one hand, for all $k \geq 1$ and almost all $t$,

$$
\begin{aligned}
\mu_{N}\{|u(t)| \geq k\} & \leq \int_{\Omega} \frac{\left|T_{k} u(t)\right|^{p^{*}}}{k^{p^{*}}} \\
& \leq \frac{C}{k^{p^{*}}}\left(\int_{\Omega}\left|\nabla T_{k} u(t)\right|^{p}\right)^{\frac{N}{N-p}} \leq C \frac{g(t)^{\frac{N}{N-p}}}{k^{N(p-1-\delta) /(N-p)}},
\end{aligned}
$$

and, on the other hand, for all $k \leq 1$ and almost all $t$,

$$
\begin{aligned}
\mu_{N}\{|u(t)| \geq k\} & \leq \int_{\Omega} \frac{\left|T_{k} u(t)\right|^{p^{*}}}{k^{p^{*}}} \\
& \leq \frac{C}{k^{p^{*}}}\left(\int_{\Omega}\left|\nabla T_{k} u(t)\right|^{p}\right)^{\frac{N}{N-p}} \leq C \frac{g(t)^{\frac{N}{N-p}}}{k^{N(p-1+\delta) /(N-p)}},
\end{aligned}
$$

from where the result follows. 
Proposition 2.7. Let $\delta \in] 0, p-1[$. Then there exists a constant $C>0$ such that for almost all $t \in[0, T]$,

$$
\begin{gathered}
\sup _{h \geq g(t)^{1 / p} M^{-1 / p}} h \mu_{N}\{|\nabla u(t)|>h\}^{\frac{2+\delta}{p}} \leq(C g(t))^{\frac{1}{p}} . \\
\sup _{h \geq g(t)^{-1 /(N-p)}} h \mu_{N}\{|\nabla u(t)|>h\}^{\frac{N-1-\delta}{N(p-1-\delta)}} \leq(C g(t))^{\frac{1}{p-1-\delta}} . \\
\sup _{h \leq g(t)^{1 / p} M^{-1 / p}} h \mu_{N}\{|\nabla u(t)|>h\}^{\frac{2-\delta}{p}} \leq(C g(t))^{\frac{1}{p}} . \\
\sup _{h \leq g(t)^{-1 /(N-p)}} h \mu_{N}\{|\nabla u(t)|>h\}^{\frac{N-1+\delta}{N(p-1+\delta)}} \leq(C g(t))^{\frac{1}{p-1+\delta}} .
\end{gathered}
$$

Proof: A similar argument to the one in [3, Lemma 4.2] can be applied. We just prove (1) and (2), since the other assertions can be proved in an analogous manner.

Before showing (1), let us point out that the operator associated with the elliptic term $-\operatorname{div} \mathbf{a}_{p}(x, \nabla u)+$ the Dirichlet boundary condition, is accretive in $L^{1}(\Omega)$ (see [3, Theorem 7.1] or [1] ); thus, $\|u(t)\|_{1} \leq\left\|u_{0}\right\|_{1}+$ $\|f\|_{1}$ for all $t \in[0, T]$ and so

$$
\mu_{N}\{|u(t)| \geq k\} \leq \frac{1}{k} \int_{\Omega}|u(t)| \leq \frac{1}{k}\left(\int_{\Omega}\left|u_{0}\right|+\int_{0}^{T} \int_{\Omega}|f|\right)=\frac{M}{k} .
$$

This fact and Proposition 2.5 imply the following inequalities.

$$
\begin{aligned}
\mu_{N}\{|\nabla u(t)|>h\} & \leq \mu_{N}\left\{\left|\nabla u(t)-\nabla T_{k} u(t)\right|>h / 2\right\}+\mu_{N}\left\{\left|\nabla T_{k} u(t)\right|>h / 2\right\} \\
& \leq \mu_{N}\{|u(t)| \geq k\}+2^{p} \int_{\Omega} \frac{\left|\nabla T_{k} u(t)\right|^{p}}{h^{p}} \leq \frac{M}{k}+2^{p} \frac{k^{1+\delta} g(t)}{h^{p}} .
\end{aligned}
$$

By taking $k=\left(\frac{h^{p} M}{g(t)}\right)^{1 /(2+\delta)} \geq 1$, we get

$$
\mu_{N}\{|\nabla u(t)|>h\} \leq\left(C h^{-p} g(t)\right)^{1 /(2+\delta)},
$$

and (1) follows.

To show (2), apply Proposition 2.6 (1) to obtain $k^{p-1-\delta} \mu_{N}\{|u(t)| \geq$ $k\}^{\frac{N-p}{N}} \leq C g(t)$ for all $k \geq 1$. Then, using Proposition 2.5,

$$
\begin{aligned}
\mu_{N}\{|\nabla u(t)|>h\} \leq \mu_{N}\{|u(t)| \geq & k\}+2^{p} \int_{\Omega} \frac{\left|\nabla T_{k} u(t)\right|^{p}}{h^{p}} \\
& \leq C \frac{g(t)^{\frac{N}{N-p}}}{k^{N(p-1-\delta) /(N-p)}}+C \frac{k^{1+\delta} g(t)}{h^{p}} .
\end{aligned}
$$

Taking $k=g(t)^{1 /(N-1-\delta)} h^{(N-p) /(N-1-\delta)} \geq 1,(2)$ is obtained. 


\section{Case $\Omega$ bounded}

Theorem 3.1. If $\frac{2 N}{N+1}<p<N$, then

$$
\begin{aligned}
u \in L^{r}\left(0, T ; \mathcal{M}^{q}(\Omega)\right) \quad \text { for } 1<q< & \frac{N(p-1)}{N-p} \\
& \text { and } r<\frac{(N+1) p-2 N}{N} \frac{q}{q-1} .
\end{aligned}
$$

Proof: Let $\frac{2 N}{N+1}<p<N$ and $q$ be as above and take $\delta>0$ such that $p>\frac{N(2+\delta)}{N+1}$ and $1<q<\frac{N(p-1-\delta)}{N-p}$. It will be enough to show that $u \in L^{r}\left(0, T ; \mathcal{M}^{q}(\Omega)\right)$ for $r=\frac{p(N+1)-N(2+\delta)}{N} \frac{q}{q-1}$.

First observe that the above inequalities imply that $r>p-1-\delta$; thus, for $k \geq 1$, we obtain that

$$
\begin{aligned}
& k^{r} \mu_{N}\{|u(t)| \geq k\}^{r / q} \\
= & \left(k^{p-1-\delta} \mu_{N}\{|u(t)| \geq k\}^{\frac{N-p}{N}}\right) \cdot\left(k^{r-p+1+\delta} \mu_{N}\{|u(t)| \geq k\}^{r-p+1+\delta}\right) .
\end{aligned}
$$

On the one hand, applying Proposition 2.6, $k^{p-1-\delta} \mu_{N}\{|u(t)| \geq k\}^{\frac{N-p}{N}} \leq$ $C g(t)$ and on the other hand, by $(2.2), k \mu_{N}\{|u(t)| \geq k\} \leq M$, so that

$$
\sup _{k \geq 1} k^{r} \mu_{N}\{|u(t)| \geq k\}^{r / q} \leq C M^{r-p+1+\delta} g(t),
$$

$g$ being an integrable function.

Since we also have, for $0<k \leq 1$, that $k^{r} \mu_{N}\{|u(t)| \geq k\}^{r / q} \leq$ $\mu_{N}\{\Omega\}^{r / q}$; the function defined by $[u(t)]_{q}^{r}$ is integrable and consequently $u \in L^{r}\left(0, T ; \mathcal{M}^{q}(\Omega)\right)$.

\section{Theorem 3.2.}

(1) If $\frac{2 N}{N+1}<p<N$, then

$$
\begin{aligned}
&|\nabla u| \in L^{r}\left(0, T ; \mathcal{M}^{q}(\Omega)\right) \quad \text { for } \frac{p}{2}<q< \frac{N(p-1)}{N-1} \\
& \quad \text { and } r<q \frac{(N+1) p-2 N}{(N+1) q-N}, \\
& \text { for } q=\frac{p}{2} \quad \text { and } r<p, \quad \text { and } \\
& \text { for } q<\frac{p}{2} \quad \text { and } r=p .
\end{aligned}
$$

(2) If $1<p \leq \frac{2 N}{N+1}$, then

$|\nabla u| \in L^{p}\left(0, T ; \mathcal{M}^{q}(\Omega)\right) \quad$ for $q<\frac{p}{2}$. 
Proof: Let $\frac{2 N}{N+1}<p<N$ and $\frac{p}{2}<q<\frac{N(p-1)}{N-1}$, and fix $\delta>0$ such that $\frac{p}{2+\delta}<q<\frac{N(p-1-\delta)}{N-1-\delta}$. We are going to show that $|\nabla u| \in L^{r}\left(0, T ; \mathcal{M}^{q}(\Omega)\right)$ for $r=q \frac{(N+1) p-N(2+\delta)}{(N+1) q-N}$, then the first part of (1) will be proved.

Since $\frac{p}{2+\delta}<q<\frac{N(p-1-\delta)}{N-1-\delta}$, let $\left.\alpha \in\right] 0,1\left[\right.$ such that $\frac{1}{q}=\frac{\alpha(N-1-\delta)}{N(p-1-\delta)}+$ $\frac{(1-\alpha)(2+\delta)}{p}$. By Proposition 2.7, for each $h \geq\left(M^{-1} g(t)\right)^{1 / p} \geq g(t)^{-1 /(N-p)}$, we obtain that

$$
\begin{aligned}
& h \mu_{N}\{|\nabla u(t)|>h\}^{1 / q} \\
& \quad=\left(h^{\alpha} \mu_{N}\{|\nabla u(t)|>h\}^{\frac{\alpha(N-1-\delta)}{N(p-1-\delta)}}\right) \cdot\left(h^{1-\alpha} \mu_{N}\{|\nabla u(t)|>h\}^{\frac{(1-\alpha)(2+\delta)}{p}}\right) \\
& \quad \leq(C g(t))^{\frac{\alpha}{p-1-\delta}+\frac{1-\alpha}{p}} .
\end{aligned}
$$

Since $r$ satisfies $\frac{1}{r}=\frac{\alpha}{p-1-\delta}+\frac{(1-\alpha)}{p}$, it follows that

$$
\sup _{h \geq M^{-1 / p} g(t)^{1 / p}} h \mu_{N}\{|\nabla u(t)|>h\}^{1 / q} \leq(C g(t))^{\frac{1}{r}} .
$$

Hence, $\sup _{h \geq M^{-1 / p} g(t)^{1 / p}} h \mu_{N}\{|\nabla u(t)|>k\}^{1 / q}$ defines a function in $L^{r}(0, T)$.

On the other hand,

$$
\sup _{0<h \leq M^{-1 / p} g(t)^{1 / p}} h \mu_{N}\{|\nabla u(t)|>h\}^{1 / q} \leq\left(M^{-1} g(t)\right)^{\frac{1}{p}} \mu_{N}\{\Omega\}^{1 / q},
$$

and thus it defines a function in $L^{p}(0, T)$. It follows from $\frac{2 N}{N+1}<p$ and $p / 2<q$ that $(N+1) q-N>0$, so that $r<p$. Therefore, $[|\nabla u(t)|]_{q}$ defines a function in $L^{r}(0, T)$ and consequently $|\nabla u| \in L^{r}\left(0, T ; \mathcal{M}^{q}(\Omega)\right)$.

Next, let $1<p<N$ and $0<q<\frac{p}{2}$. Fix $\delta>0$ such that $0<q<\frac{p}{2+\delta}$. We are going to see that

$$
|\nabla u| \in L^{p}\left(0, T ; \mathcal{M}^{\frac{p}{2+\delta}}(\Omega)\right) ;
$$

this will prove the second part of (1), and (2).

By Proposition 2.7 (1),

$$
\sup _{h \geq g(t)^{1 / p} M^{-1 / p}} h \mu_{N}\{|\nabla u(t)|>h\}^{\frac{2+\delta}{p}} \leq(C g(t))^{\frac{1}{p}}
$$

and obviously

$$
\sup _{0<h \leq g(t)^{1 / p} M^{-1 / p}} h \mu_{N}\{|\nabla u(t)|>h\}^{\frac{2+\delta}{p}} \leq\left(M^{-1} g(t)\right)^{\frac{1}{p}} \mu_{N}\{\Omega\}^{\frac{2+\delta}{p}} .
$$


Thus, $\sup _{h>0} h \mu_{N}\{|\nabla u(t)|>k\}^{\frac{2+\delta}{p}}$ defines a function in $L^{p}(0, T)$ and

$$
|\nabla u| \in L^{p}\left(0, T ; \mathcal{M}^{\frac{p}{2+\delta}}(\Omega)\right) .
$$

Finally, the case $q=\frac{p}{2}, r<p$, follows directly from embedding results in Marcinkiewicz spaces.

\section{Case $\Omega$ unbounded}

Theorem 4.1. If $p \neq \frac{2 N}{N+1}$, then $u \in L^{r}\left(0, T ; \mathcal{M}^{q}(\Omega)\right)$ for $q$ strictly between 1 and $\frac{N(p-1)}{N-p}$, and $r<\frac{(N+1) p-2 N}{N} \frac{q}{q-1}$.

Proof: Assume first that $\frac{2 N}{N+1}<p<N$; let $1<q<\frac{N(p-1)}{N-p}$ and fix $\delta>0$ such that $1<q<\frac{N(p-1-\delta)}{N-p}$. We are going to prove that $u \in L^{r}\left(0, T ; \mathcal{M}^{q}(\Omega)\right)$ for $r=\frac{N(p-1-\delta)-(N-p)}{N} \frac{q}{q-1}$. Let $\left.\alpha_{ \pm} \in\right] 0,1[$ such that $\frac{1}{q}=\alpha_{ \pm}+\frac{\left(1-\alpha_{ \pm}\right)(N-p)}{N(p-1 \pm \delta)}$. By Proposition 2.6 and (2.2), we obtain that

$$
\begin{aligned}
& \sup _{k \geq 1} k \mu_{N}\{|u(t)|>k\}^{1 / q} \leq M^{\alpha_{-}}(C g(t))^{\frac{1-\alpha_{-}}{p-1-\delta}}, \\
& \sup _{k \leq 1} k \mu_{N}\{|u(t)|>k\}^{1 / q} \leq M^{\alpha_{+}}(C g(t))^{\frac{1-\alpha_{+}}{p-1+\delta}},
\end{aligned}
$$

and thus $\sup _{k>0} k \mu_{N}\{|u(t)|>k\}^{1 / q}$ defines a function that belongs to $L^{r}(0, T)$, with $r$ the minimun of $\frac{N(p-1-\delta)}{\left(1-\alpha_{-}\right)(N-p)}$ and $\frac{N(p-1+\delta)}{\left(1-\alpha_{+}\right)(N-p)}$, that is,

$$
r=\frac{N(p-1-\delta)-(N-p)}{N} \frac{q}{q-1} .
$$

In the case $1<p<\frac{2 N}{N+1}$, consider $\delta>0$ small enough such that $\frac{N(p-1+\delta)}{N-p}<q<1$. Reasoning similarly one shows that $u \in L^{r}\left(0, T ; \mathcal{M}^{q}(\Omega)\right)$ for $r=\frac{N(p-1+\delta)-(N-p)}{N} \frac{q}{q-1}$.

Theorem 4.2. If $p \neq \frac{2 N}{N+1}$, then $|\nabla u| \in L^{r}\left(0, T ; \mathcal{M}^{q}(\Omega)\right)$ for $q$ strictly between $\frac{p}{2}$ and $\frac{N(p-1)}{N-1}$, and $r<q \frac{(N+1) p-2 N}{(N+1) q-N}$. 
Proof: Let us show it for $1<p<\frac{2 N}{N+1}$. The other case follows in the same way. Let $\frac{N(p-1)}{N-1}<q<\frac{p}{2}$ and fix $\delta>0$ such that $\frac{N(p-1+\delta)}{N-1+\delta}<$ $q<\frac{p}{2+\delta}$. We are going to prove that $|\nabla u| \in L^{r}\left(0, T ; \mathcal{M}^{q}(\Omega)\right)$ for $r=$ $q \frac{N(2-\delta)-p(N+1)}{N-q(N+1)}$. Since $\frac{N(p-1+\delta)}{N-1+\delta}<q<\frac{p}{2+\delta}<\frac{p}{2-\delta}$, let $\left.\alpha \in\right] 0,1[$ such that $\frac{1}{q}=\frac{\alpha(N-1+\delta)}{N(p-1+\delta)}+\frac{(1-\alpha)(2-\delta)}{p}$. By (3) and (4) in Proposition 2.7, we obtain that

$$
\sup _{0<h \leq g(t)^{-1 /(N-p)}} h \mu_{N}\{|\nabla u(t)|>k\}^{1 / q} \leq(C g(t))^{\frac{\alpha}{p-1+\delta}+\frac{1-\alpha}{p}}
$$

and thus $\sup _{h \leq g(t)^{-1 /(N-p)}} h \mu_{N}\{|\nabla u(t)|>k\}^{1 / q}$ defines a function in $L^{r_{1}}(0, T)$, with $r_{1}=q \frac{N(2-\delta)-p(N+1)}{N-q(N+1)}$ (i.e., the number that satisfies $\left.\frac{1}{r_{1}}=\frac{\alpha}{p-1+\delta}+\frac{(1-\alpha)}{p}\right)$. Analogously, the function defined by $\sup _{g(t)^{-1 /(N-p)} \leq h \leq\left(M^{-1} g(t)\right)^{1 / p}} h \mu_{N}\{|\nabla u(t)|>k\}^{1 / q}$ belongs to $L^{r_{2}}(0, T)$, where $r_{2}=q \frac{N(1+\delta)(2-\delta)-p(N(1-\delta)+(1+\delta))}{N(1+\delta)-q(N(1-\delta)+(1+\delta))}$, and the function defined by $\sup _{h>\left(M^{-1} g(t)\right)^{1 / p}} h \mu_{N}\{|\nabla u(t)|>k\}^{1 / q}$ belongs to $L^{r_{3}}(0, T)$, where $r_{3}=$ $q \frac{N(2+\delta)-p(N+1)}{N-q(N+1)}$. Therefore, the function defined by $[|\nabla u(t)|]_{q}$ belongs to $L^{r}(0, T)$, where $r=\min \left\{r_{1}, r_{2}, r_{3}\right\}=q \frac{N(2-\delta)-p(N+1)}{N-q(N+1)}$.

\section{Other estimates}

To finish this note we show what happens when we take into account another estimate instead of that of Proposition 2.5.

\section{1. $p$-Laplacian evolution equations.}

For $p$-Laplacian evolution equations and $f=0$, the results of $[\mathbf{1}]$ and [4] imply that, for any $0<\alpha<1$,

$$
\int_{0}^{T} \sup _{k>0}\left(\int_{\Omega} \frac{\left|\nabla T_{k} u(t)\right|^{p}}{k}\right)^{\alpha}<\infty
$$

Indeed, let $k>0$; by [1] , if $u_{0} \in D(A)$, then the entropy solution belongs to $W^{1,1}\left(0, T ; L^{1}(\Omega)\right)$ and, for almost all $t$,

$$
\frac{1}{k} \int_{\Omega}\left\langle\mathbf{a}_{p}(x, \nabla u(t)), \nabla T_{k} u(t)\right\rangle \leq-\int_{\Omega} u^{\prime}(t) \frac{T_{k} u(t)}{k} .
$$


Then $\int_{\Omega} \frac{\left|\nabla T_{k} u(t)\right|^{p}}{k} \leq \int_{\Omega}\left|u^{\prime}(t)\right|$. Since, when $p$-Laplacian is considered, $\left\|u^{\prime}(t)\right\|_{1} \leq \frac{\left\|u_{0}\right\|_{1}}{t}$ (see [4]), it yields $\int_{\Omega} \frac{\left|\nabla T_{k} u(t)\right|^{p}}{k} \leq \frac{\left\|u_{0}\right\|_{1}}{t}$. Now it follows from $\overline{D(A)}=L^{1}(\Omega)$ and the continuous dependence on the data that the inequality $\int_{\Omega} \frac{\left|\nabla T_{k} u(t)\right|^{p}}{k} \leq \frac{\left\|u_{0}\right\|_{1}}{t}$ holds a.e. for every $u_{0} \in L^{1}(\Omega)$ and every $k>0$. Therefore, (5.1) holds.

Taking this estimate and following the steps given in this paper, one will obtain, besides Theorem 1 and 2, the following improvement.

For any $1<p<N$,

$$
\begin{aligned}
u & \in L^{r}\left(0, T ; \mathcal{M}^{\frac{N(p-1)}{N-p}}(\Omega)\right) & & \text { for } r<p-1 . \\
|\nabla u| & \in L^{r}\left(0, T ; \mathcal{M}^{\frac{N(p-1)}{N-1}}(\Omega)\right) & & \text { for } r<p-1 . \\
|\nabla u| & \in L^{r}\left(0, T ; \mathcal{M}^{\frac{p}{2}}(\Omega)\right) & & \text { for } r<p .
\end{aligned}
$$

Let us point out that, in particular, one has $u \in L^{r}\left(0, T ; \mathcal{M}^{\frac{N(p-1)}{N-p}}(\Omega)\right)$ and $|\nabla u| \in L^{r}\left(0, T ; \mathcal{M}^{\frac{N(p-1)}{N-1}}(\Omega)\right)$ for any $r<p-1$, so that this argument gives us the same type of regularity obtained in $[\mathbf{3}]$ for the stationary problem associated to $(\mathrm{P})$.

\subsection{More regular initial data.}

We point out that the summability on the solution and its derivative improves when $L^{1}$-data are replaced by more regular ones (see [12] and [15] where data $u_{0} \in L^{s}(\Omega)$, with $1<s<2$, are considered). Following the arguments of the above sections, we will show what is the precise summability assuming that $f=0$ and the initial datum $u_{0}$ belongs either to a Lebesgue space or to a Marcinkiewicz one. Finally, we discuss an example which proves that these estimates are sharp.

Whether $u_{0}$ lives in $L^{s}(\Omega)$ or in $\mathcal{M}^{s}(\Omega)$, our computations imply $\|u(t)\| \leq\left\|u_{0}\right\|$ for all $t>0$. This fact holds true as a consequence of being these spaces normal and the operator associated to the elliptic term: $-\operatorname{div} \mathbf{a}_{p}(x, \nabla u)+$ Dirichlet boundary condition, completely accretive (see [5] for the definition and [3, Theorem 7.1]). In order to use the arguments of the above sections, we need another fact which is stated in the following result.

Lemma 5.1. Let $u$ be the entropy solution of $(P)$ and let $1<s<2$.

(1) If $u_{0} \in L^{s}(\Omega) \cap L^{1}(\Omega)$, then

$$
\int_{0}^{T} \sup _{k>0}\left(\int_{\Omega} \frac{\left|\nabla T_{k} u(t)\right|^{p}}{k^{2-s}}\right)<+\infty
$$


(2) If $u_{0} \in \mathcal{M}^{s}(\Omega) \cap L^{1}(\Omega)$, then for every $\delta>0$ there is $g \in L^{1}(0, T)$ such that

$$
\int_{\Omega} \frac{\left|\nabla T_{k} u(t)\right|^{p}}{k^{2-s}}= \begin{cases}k^{\delta} g(t), & \text { if } t \in[0, T] \text { and } k \geq 1 \\ k^{-\delta} g(t), & \text { if } t \in[0, T] \text { and } k \leq 1\end{cases}
$$

Proof: (1) As in the proof of Proposition 2.5, we only consider $k \geq 1$, the other case is similar.

To begin with the proof, take a measurable function $\lambda:[0, T] \rightarrow$ $\left[1,+\infty\left[\right.\right.$ and the sets $A_{j}:=\left\{t \in[0, T]: 2^{j} \leq \lambda(t)<2^{j+1}\right\}, j \geq 0$, and denote

$$
I=\int_{0}^{T} \int_{\{\lambda(t) / 2<|u(t)|<\lambda(t)\}} \frac{|\nabla u(t)|^{p}}{\lambda(t)^{2-s}} .
$$

Then

$$
\begin{aligned}
I & =\sum_{j=0}^{\infty} \int_{A_{j}} \int_{\{\lambda(t) / 2<|u(t)|<\lambda(t)\}} \frac{|\nabla u(t)|^{p}}{\lambda(t)^{2-s}} \\
& \leq \sum_{j=0}^{\infty} \frac{1}{2^{j(2-s)}} \int_{\left\{2^{j-1}<|u|<2^{j+1}\right\}}|\nabla u|^{p}
\end{aligned}
$$

and, since

$$
\int_{\left\{2^{j-1}<|u|<2^{j-1}+3 \cdot 2^{j-1}\right\}} \frac{|\nabla u|^{p}}{3 \cdot 2^{j-1}} \leq \int_{\left\{\left|u_{0}\right|>2^{j-1}\right\}}\left|u_{0}\right|
$$

for all $j \geq 0$ (see [15, Theorem 2.2], it follows that there exists $C_{1}>0$ such that

$$
\begin{aligned}
I & \leq C_{1} \sum_{j=0}^{\infty} 2^{(j-2)(s-1)} \int_{\left\{\left|u_{0}\right|>2^{j-1}\right\}}\left|u_{0}\right| \\
& =C_{1} \sum_{j=0}^{\infty} 2^{(j-2)(s-1)} \sum_{n=j}^{\infty} \int_{\left\{2^{n-1}<\left|u_{0}\right| \leq 2^{n}\right\}}\left|u_{0}\right| .
\end{aligned}
$$


Now, Fubini's Theorem implies

$$
I \leq C_{1} \sum_{n=0}^{\infty} \sum_{j=0}^{n} 2^{(j-2)(s-1)} \int_{\left\{2^{n-1}<\left|u_{0}\right| \leq 2^{n}\right\}}\left|u_{0}\right|
$$

and estimating the geometrical series, we get $C_{2}>0$ such that

$$
\begin{aligned}
I & \leq C_{2} \sum_{n=0}^{\infty} 2^{(n-1)(s-1)} \int_{\left\{2^{n-1}<\left|u_{0}\right| \leq 2^{n}\right\}}\left|u_{0}\right| \\
& \leq C_{2} \sum_{n=0}^{\infty} \int_{\left\{2^{n-1}<\left|u_{0}\right| \leq 2^{n}\right\}}\left|u_{0}\right|^{s}<+\infty .
\end{aligned}
$$

One deduces from Lemma 2.3 that

$$
\int_{0}^{T} \sup _{k \geq 1}\left(\int_{\{k / 2<|u(t)|<k\}} \frac{|\nabla u(t)|^{p}}{k^{2-s}}\right)<+\infty .
$$

Hence,

$$
\begin{aligned}
& \int_{0}^{T} \sup _{k \geq 1}\left(\int_{\Omega} \frac{\left|T_{k} \nabla u(t)\right|^{p}}{k^{2-s}}\right) \\
= & \int_{0}^{T} \sup _{k \geq 1}\left(\sum_{j=0}^{\infty} \frac{1}{2^{j(2-s)}} \int_{\left\{k / 2^{j+1}<|u(t)|<k / 2^{j}\right\}} \frac{|\nabla u(t)|^{p}}{\left(k / 2^{j}\right)^{2-s}}\right) \\
\leq & \sum_{j=0}^{\infty} \frac{1}{2^{j(2-s)}}\left[\int_{0}^{T} \sup _{k \geq 1}\left(\int_{\{k / 2<|u(t)|<k\}} \frac{|\nabla u(t)|^{p}}{k^{2-s}}\right)\right]
\end{aligned}
$$

and, since $\sum_{j=0}^{\infty} \frac{1}{2^{j(2-s)}}$ converges, it yields

$$
\int_{0}^{T} \sup _{k \geq 1}\left(\int_{\Omega} \frac{\left|T_{k} \nabla u(t)\right|^{p}}{k^{2-s}}\right)<+\infty .
$$

The proof of (2) is analogous.

Using the arguments of the above sections, from Lemma 5.1 the following results yield. 
Theorem 5.2. Let $u$ be the entropy solution of $(P)$ and let $1<s<2$.

(1) If $u_{0} \in L^{s}(\Omega) \cap L^{1}(\Omega)$, then $u \in L^{r}\left(0, T ; \mathcal{M}^{q}(\Omega)\right)$, where $r \leq$ $q \frac{(N+s) p-2 N}{N(q-s)}$ and $s<q \leq \frac{N(p+s-2)}{N-p}$, and $|\nabla u| \in L^{r}\left(0, T ; \mathcal{M}^{q}(\Omega)\right)$, where $r \leq q \frac{(N+s) p-2 N}{(N+s) q-s N}$ and $q$ lies between $\frac{N(p+s-2)}{N+s-2}$ and $\frac{s p}{2}$.

(2) If $u_{0} \in \mathcal{M}^{s}(\Omega) \cap L^{1}(\Omega)$, then $u \in L^{r}\left(0, T ; \mathcal{M}^{q}(\Omega)\right)$, where $r<$ $q \frac{(N+s) p-2 N}{N(q-s)}$ and $s<q<\frac{N(p+s-2)}{N-p}$, and $|\nabla u| \in L^{r}\left(0, T ; \mathcal{M}^{q}(\Omega)\right)$, for $q$ strictly between $\frac{N(p+s-2)}{N+s-2}$ and $\frac{s p}{2}$, and $r<q \frac{(N+s) p-2 N}{(N+s) q-s N}$.

To finish this paper, we prove that the above estimates are sharp in the framework of Marcinkiewicz spaces. We will show that, in the case $p=2$ and $u_{0} \in \mathcal{M}^{s}(\Omega) \cap L^{1}(\Omega)$, we have $u \in L^{r}\left(0, T ; \mathcal{M}^{q}(\Omega)\right)$, with $r<q \frac{2 s}{N(q-s)}$ and $s<q<\frac{N s}{N-p}$, but $u \notin L^{r}\left(0, T ; \mathcal{M}^{q}(\Omega)\right)$, with $r=q \frac{2 s}{N(q-s)}$ and $s<q<\frac{N s}{N-p}$.

Example 5.3. Let us consider $\Omega=\mathbb{R}^{N}$ and the problem of finding $u \in C\left([0, T], L^{1}(\Omega)\right)$ such that

$$
\begin{cases}u_{t}-\Delta u=0, & \text { in }] 0, T\left[\times \mathbb{R}^{N} ;\right. \\ u(0, .)=u_{0}, & \text { in } \mathbb{R}^{N} ;\end{cases}
$$

where $u_{0}(x)=\frac{1}{|x|^{\alpha}} \chi_{B(0,1)}(x)$, with $N / 2<\alpha<N$. Denoting $s=N / \alpha$, it is straighforward that $1<s<2$ and $u_{0} \in \mathcal{M}^{s}\left(\mathbb{R}^{N}\right) \cap L^{1}\left(\mathbb{R}^{N}\right)$, but $u_{0} \notin L^{s}\left(\mathbb{R}^{N}\right)$. We know that the solution of this linear problem is given by

$$
u(t, x)=\frac{1}{(4 \pi t)^{N / 2}} \int_{\mathbb{R}^{N}} e^{\frac{-|x-y|^{2}}{4 t}} u_{0}(y) d y .
$$

Changing variables: $x=\eta t^{t / 2}$ and $y=\xi t^{t / 2}$, we obtain

$$
\begin{aligned}
& \int_{0}^{T}[u(t)]_{q}^{r} d t \\
& =\int_{0}^{T} \sup _{k>0} k^{r} \mu\left\{x \in \mathbb{R}^{N}:|u(t, x)|>k\right\}^{r / q} d t \\
& =\int_{0}^{T} \sup _{k>0} k^{r}\left(\int_{\mathbb{R}^{N}} \chi_{\left\{1 /(4 \pi)^{N / 2} \int_{\mathbb{R}^{N}} e^{\frac{-|x-y|^{2}}{4 t}} u_{0}(y) d y>k t^{N / 2}\right\}}(x) d x\right)^{r / q} d t \\
& =\int_{0}^{T} t^{\frac{N r}{2 q}} \sup _{k>0} k^{r}\left(\int_{\mathbb{R}^{N}} \chi_{\left\{1 /(4 \pi)^{N / 2} \int_{\mathbb{R}^{N}} e^{\frac{-|\eta-\xi|^{2}}{4}} u_{0}\left(\xi t^{1 / 2}\right) d \xi>k\right\}}(\eta) d \eta\right)^{r / q} d t \\
& =\int_{0}^{T} t^{\frac{N r}{2 q}} \sup _{k>0} k^{r} \mu\left\{\eta \in \mathbb{R}^{N}: \frac{1}{(4 \pi)^{N / 2}} \int_{B\left(0, t^{-1 / 2}\right)} \frac{e^{\frac{-|\eta-\xi|^{2}}{4}}}{|\xi|^{\alpha}} d \xi>k t^{\alpha / 2}\right\}^{r / q} d t .
\end{aligned}
$$


By denoting now $h=k t^{1 / 2}$ and $g(t, \eta)=\frac{1}{(4 \pi)^{N / 2}} \int_{B\left(0, t^{-1 / 2}\right)} \frac{e^{\frac{-|\eta-\xi|^{2}}{4}}}{|\xi|^{\alpha}} d \xi$, we get

$$
\int_{0}^{T}[u(t)]_{q}^{r} d t=\int_{0}^{T} t^{\frac{r}{2}\left(\frac{N}{q}-\alpha\right)} \sup _{h>0} h^{r} \mu\left\{\eta \in \mathbb{R}^{N}: g(t, \eta)>h\right\}^{r / q} d t .
$$

Define

$$
\begin{aligned}
& g_{1}(\eta)=\frac{1}{(4 \pi)^{N / 2}} \int_{B\left(0, T^{-1 / 2}\right)} \frac{e^{\frac{-|\eta-\xi|^{2}}{4}}}{|\xi|^{\alpha}} d \xi, \\
& g_{2}(\eta)=\frac{1}{(4 \pi)^{N / 2}} \int_{\mathbb{R}^{N}} \frac{e^{\frac{-|\eta-\xi|^{2}}{4}}}{|\xi|^{\alpha}} d \xi
\end{aligned}
$$

and $c_{i}=\sup _{h>0} h^{r} \mu\left\{\eta \in \mathbb{R}^{N}: g_{i}(\eta)>h\right\}^{r / q}, i=1,2$ (observe that both constants are finite). Since $g_{1}(\eta) \leq g(t, \eta) \leq g_{2}(\eta)$ for all $t \in[0, T]$, it follows that

$$
c_{2} \int_{0}^{T} t^{\frac{r}{2}\left(\frac{N}{q}-\alpha\right)} d t \leq \int_{0}^{T}[u(t)]_{q}^{r} d t \leq c_{1} \int_{0}^{T} t^{\frac{r}{2}\left(\frac{N}{q}-\alpha\right)} d t .
$$

Thus, if $r<q \frac{2 s}{N(q-s)}$, then $\frac{r}{2}\left(\frac{N}{q}-\alpha\right)>-1$ and so $\int_{0}^{T}[u(t)]_{q}^{r} d t<$ $+\infty$; however, if $r=q \frac{2 s}{N(q-s)}$, then $\frac{r}{2}\left(\frac{N}{q}-\alpha\right)=-1$ and consequently $\int_{0}^{T}[u(t)]_{q}^{r} d t=+\infty$. Therefore, in the first case, the solution $u$ belongs to $L^{r}\left(0, T ; \mathcal{M}^{q}\left(\mathbb{R}^{N}\right)\right)$, but it is not so in the second one.

\section{References}

1. F. Andreu, J. M. Mazón, S. Segura de León and J. Toledo, Existence and uniqueness for a degenerate parabolic equation with $L^{1}$ data, Trans. Amer. Math. Soc. 351 (1999), 285-306.

2. J. Appell And P. P. Zabrejko, "Nonlinear Superposition Operators," Cambridge Univ. Press, Cambridge, 1990.

3. Ph. Bénilan, L. Boccardo, Th. Gallouët, R. Gariepy, M. Pierre and J. L. VÁzquez, An $L^{1}$ theory of existence and uniqueness of solutions of nonlinear elliptic equations, Ann. Scuola Norm. Sup. Pisa Cl. Sci. (4) 22 (1995), 241-273. 
4. Ph. Bénilan and M. G. Crandall, Regularizing effects of homogeneous evolution equations, in "Contributions to Analysis and Geometry," (D. N. Clark et al., ed.), John Hopkins Univ. Press, Baltimore, Md., 1981, pp. 23-39.

5. Ph. Bénilan and M. G. Crandall, Completely accretive operators, in "Semigroup Theory and Evolution Equations," (Ph. Clément, E. Mitidieri and B. de Pagter, ed.), Marcel Dekker Inc., New York, 1991, pp. 41-75.

6. Ph. Bénilan, M. G. Crandall and A. Pazy, "Evolution Equations Governed by Accretive Operators," forthcoming book.

7. D. Blanchard and F. Murat, Renormalized solutions of non linear parabolic problems with $L^{1}$ data: Existence and uniqueness, Proc. Roy. Soc. Edinbourgh Sect. A 127(6) (1997), 1137-1152.

8. L. Boccardo, A. Dall'Aglio, Th. Gallouët and L. Orsina, Nonlinear parabolic equations with measure data, J. Funct. Anal. 147 (1997), 237-258.

9. L. Boccardo and Th. Gallouët, Nonlinear elliptic and parabolic equations involving measure data, J. Funct. Anal. 87 (1989), 149-169.

10. J. L. Lions, "Quelques Méthodes de Résolution des Problèmes aux Limites Non Linéaires," Dunod, Gauthier-Villars, Paris, 1969.

11. A. Prignet, Existence and uniqueness of entropy solutions of parabolic problems with $L^{1}$ data, Nonlinear Anal. 28 (1997), 1943-1954.

12. A. Porretta, Regularity for entropy solutions of a class of parabolic equations with non regular initial datum, Dynam. Systems Appl. 7 (1998), 53-71.

13. J. M. Rakotoson, Generalized solutions in a type of sets for problems with measures as data, Differential Integral Equations 6 (1993), $27-36$.

14. J. M. Rakotoson, Uniqueness of renormalized solutions in a $T$-set for the $L^{1}$-data problem and the link between various formulations, Indiana Univ. Math. J. 43(2) (1994), 285-293.

15. S. Segura De LeÓn, Estimates for solutions of non-linear parabolic equations, Boll. Un. Mat. Ital. B (7) 11 (1997), 987-996.

16. X. XU, On the initial-boundary-value problem for $u_{t}-\operatorname{div}\left(|\nabla u|^{p-2}\right.$ $\nabla u)=0$, Arch. Rational Mech. Anal. 127 (1994), 319-335. 
17. W. P. Ziemer, "Weakly Differentiable Functions," Springer-Verlag, New York, 1989.

\author{
Departament d'Anàlisi Matemàtica \\ Universitat de València \\ Dr. Moliner 50 \\ 46100 Burjassot \\ València \\ SPAIN \\ e-mail: Sergio.Segura@uv.es \\ e-mail: Jose.Toledo@uv.es
}

Primera versió rebuda el 22 d'octubre de 1998, darrera versió rebuda el 6 de setembre de 1999 